\title{
Globe
}

Revue internationale d'études québécoises

\section{Janusz Przychodzen (dir.), Écritures québécoises, inspirations orientales. Dialogues réinventés ?, Québec, Presses de l’Université Laval, 2013}

\section{Camille Arpin}

Volume 17, numéro 1, 2014

URI : https://id.erudit.org/iderudit/1028647ar

DOI : https://doi.org/10.7202/1028647ar

Aller au sommaire du numéro

Éditeur(s)

Globe, Revue internationale d'études québécoises

ISSN

1481-5869 (imprimé)

1923-8231 (numérique)

Découvrir la revue

Citer ce compte rendu

Arpin, C. (2014). Compte rendu de [Janusz Przychodzen (dir.), Écritures québécoises, inspirations orientales. Dialogues réinventés ?, Québec, Presses de l’Université Laval, 2013]. Globe, 17(1), 248-251.

https://doi.org/10.7202/1028647ar d'utilisation que vous pouvez consulter en ligne.

https://apropos.erudit.org/fr/usagers/politique-dutilisation/ 
politique, pourtant fondamental pour apprécier toute la complexité des relations entre anglophones et francophones dans les années 1960. Des ouvrages tels Un pays à refaire de Kenneth McRoberts ou encore Trudeau et la fin d'un rêve canadien de Guy Laforest auraient été des ajouts judicieux à la bibliographie. Cela aurait sans doute permis d'éviter quelques erreurs, notamment lorsque l'auteure mentionne que Frank Scott s'est retrouvé isolé à la commission Laurendeau-Dunton devant les positions de Paul Lacoste, alors que c'est plutôt le contraire qui s'est produit (p. 144).

Valérie Lapointe Gagnon

Université York

\section{Janusz Przychodzen (dir.) \\ Écritures québécoises, inspirations orientales. Dialogues réin- ventés?, Québec, Presses de l'Université Laval, 2013.}

Janusz Przychodzen, professeur agrégé au Département d'études françaises de l'Université York, souhaite réorienter, au moyen de son plus récent ouvrage, la conception de l'Orient qui est véhiculée dans la littérature québécoise. Il désire s'éloigner de celle instaurée par Edward Saïd depuis la publication de son livre Orientalism en 1978. Il n'y est plus question d'une vision dichotomique où l'altérité est au premier plan, comme Przychodzen l'a précédemment étudiée dans son ouvrage Asie du soi, Asie de l'autre. Récits et figures de l'altérité. Cette fois-ci, il s'agit de comprendre l'orientalisme dans la littérature québécoise comme une multiplicité de voix qui permettent d'accéder à un nouveau dialogue, dont les assises sont d'ordre esthétique et poétique. Pour parvenir à circonscrire ce dialogue, l'auteur a sélectionné comme angles d'approche l'intersubjectivité, l'interculturalité et la transculturalité, qui, à leur façon, éliminent les idées préconçues et créent une situation d'échange positive. Les textes de ce recueil ont été subdivisés en quatre grandes thématiques: le langage, les lieux de paroles, les stéréotypes et l'Histoire.

Dans le premier chapitre, "Entre violence et silence : langage", Michel Peterson et Carmen Mata Barreiro examinent comment les œuvres de Wajdi Mouawad et d'Abla Farhoud utilisent d'autres formes de langage pour donner sens à leurs récits. Chez Mouawad, la langue qui permet de 
communiquer est celle du Walten, terme emprunté à Heidegger, une violence sous-jacente constitutive et traumatisante qui, lorsqu'on l'affronte, permet d'effectuer un retour au pays d'origine. Il ne s'agit pas du Liban de Mouawad, mais plutôt d'un voyage «à travers les différentes couches géologiques de la psyché » (p. 13). Pour Farhoud, le langage passe par différentes variations du silence. Barreiro en répertorie trois : le silence de l'incompréhension et de l'incommunicabilité, qui résulte d'une difficulté à s'intégrer à une nouvelle culture; le silence reconstructeur, qui devient un espace et un temps de réflexion durant un processus de deuil ; et, finalement, le silence de l'impossibilité, qui résulte de l'impuissance des mots à communiquer les horreurs de la guerre. En somme, lorsque la parole ne peut traduire convenablement toutes les situations, il est préférable d'utiliser des langages universels comme la violence et le silence.

Le deuxième chapitre, "Nouveaux lieux de parole ", étudie les places occupées par la parole et l'écriture chez trois auteurs, Nadine Ltaif, Kim Thùy et Ook Chung. Les écrits de Nadine Ltaif, souligne Lucie Lequin, lui permettent de se mouvoir dans "un lieu de réinterprétations multiples" (p. 55). Ce lieu, sans frontières, propose un dialogue sans contraintes en s'éloignant des maux occasionnés par la guerre pour accéder à un monde d'espoir et de beauté. Grâce à l'écriture, Ltaif peut adopter une appartenance plurielle. Anne Caumartin soutient que le récit de Kim Thùy, $R u$, est une «entreprise de cohésion» (p. 77) où la mémoire devient un lieu de référence pour l'Orient. Cet espace n'est pas le lieu du ressentiment et du déchirement d'une identité perdue, mais plutôt un endroit où l'identité d'un sujet responsable est en train de se construire dans un futur commun. Puis, chez Ook Chung, Catherine P. Cua explique comment cet auteur, depuis un dialogue entre cultures, brise les frontières littéraires en créant " un ouvrage polyphonique et hybride tant dans sa forme que dans son contenu» (p. 87). Dans L'expérience interdite, la multiplicité des personnages met en évidence l'illusion d'un dialogue, qui relève plutôt d'une seule voix polyphonique. Le dialogue ne s'effectue plus au niveau de la voix narrative, mais entre les différents fragments génériques qui créent une œuvre plurielle originale. Ces trois textes permettent de reconnaître un désir d'affranchissement, un désir de se mouvoir dans un espace libre de toute catégorisation et où l'avenir est à construire.

Les deux premiers chapitres de l'ouvrage dirigé par Przychodzen suivaient une ligne de pensée relativement nette, les nouvelles formes de langage littéraire permettant d'accéder à de nouveaux lieux où ces langages prennent place. Or le troisième chapitre, "Que peut le stéréotype?», brise 
quelque peu le fil argumentatif de l'ouvrage, délaissant la portée poétique et esthétique des œuvres étudiées. Le texte de Ching Selao explique comment l'utilisation du stéréotype orientaliste par Dany Laferrière permet de repenser l'altérité. Les clichés du Japon (un Japon érotisé par la femme et un Japon guerrier signe de mort) marquent l'ignorance de l'auteur sur ce pays. Toutefois, cette ignorance est signe de liberté, car le Japon mis en scène par Laferrière lui appartient en ce qu'il l'invente. Le cliché confronte les imaginaires occidentaux et orientaux, car il met en évidence que l'exploration de l'autre se fait sans quitter ce que l'on connait. L'utilisation du stéréotype par Laferrière repense l'acte de création littéraire et annihile l'altérité de l'autre puisqu'elle se retrouve dans le cliché. Le texte suivant, écrit par Rachel Bouvet, étudie dans les récits de voyage de deux auteurs québécois, Eugène Cloutier et Jacques Hébert, le rapport qu'ils entretiennent avec l'Afrique du Nord. Elle définit le contact direct que Cloutier a avec la communauté maghrébine comme étant une altérité lointaine. Chez Hébert, le contact avec la communauté arabe passe par une médiation, un intermédiaire européen. Il s'agit d'une altérité proximale. La rencontre de l'autre crée une perte de repères qui permet de dépasser les idées préconçues.

Dans le dernier chapitre, "Le poids de l'Histoire», Yatyn Sahye démontre que Le dieu dansant de Yolande Villemaire est un roman historique postmoderne par le truchement des agissements des personnages. Le roman de Villemaire, suggère-t-on au final, permet au lecteur d'adopter « un monde de nuances et de vérités plurielles» (p. 150) et d'abandonner une vérité assimilatrice, totalisante et stérilisante - ce n'est donc qu'à la toute fin de l'article que l'on saisit comment le postmodernisme historique se lie aux autres textes de l'ouvrage. Celui-ci se clôt sur l'article de Janusz Przychodzen portant sur le Triptyque des temps perdus de Jean Marcel. Dans un argumentaire long et parfois moins limpide, Przychodzen étudie la poétique de Marcel et son utilisation de l'Histoire comme un héritage culturel qui peut être modifié, contesté et imaginé. Dans sa trilogie, Marcel repense l'origine : il n'est pas question d'identité, mais d'unité. Il s'agit d'une «écriture qui postule une dissociation de la représentation avec l'objet représenté sans pour autant se dénier le droit même de représenter" (p. 169). Ainsi, l'Histoire s'écrit sous des formes originales qui lui permettent de s'émanciper de la vision figée du sacré.

En somme, le livre de Janusz Przychodzen, Écritures québécoises, inspirations orientales. Dialogues réinventés?, développe d'intéressantes nouvelles pistes pour penser l'orientalisme dans la littérature québécoise. S'il nous apparaît manquer de cohérence entre les textes des deux derniers 
chapitres, il s'agit d'un dialogue pertinent et actuel sur la littérature québécoise contemporaine, dont Przychodzen a bien su circonscrire les différentes orientations.

Camille Arpin

Université Laval

\section{María Sierra Córdoba Serrano \\ Le Québec traduit en Espagne. Analyse sociologique de l'exportation d'une culture périphérique, Ottawa, Presses de l'Université d'Ottawa, 2013.}

La visibilité des écrivains du Québec à l'étranger résulte rarement du hasard ou d'un engouement spontané. Dans bien des cas, cette présence québécoise dans d'autres cultures est au contraire le résultat d'efforts concertés, de stratégies de réseautage et de subventions ciblées pour favoriser des échanges culturels entre de nombreux partenaires: dans ce cas-ci, les maisons d'édition, les États concernés et les publics. C'est précisément ce que démontre le premier livre (dérivé d'une thèse de doctorat soutenue à l'Université d'Ottawa) de María Sierra Córdoba Serrano, qui enseigne au Monterey Institute of International Studies, en Californie. Le but de son livre est de montrer comment des dizaines de livres québécois ont été traduits et édités en Espagne, principalement en Catalogne. Le corpus choisi de nos auteurs traduits en castillan ou en catalan est impressionnant par son ampleur et souvent insoupçonné : des œuvres de Nelly Arcan, d'Yves Beauchemin, de Ying Chen, de Dominique Demers, de Cécile Gagnon (14 ouvrages), d'Anne Hébert, de Louis Hémon (Maria Chapdelaine) et de plusieurs autres, totalisant 77 œuvres, principalement des romans et des ouvrages pour la jeunesse. Une attention particulière est ici accordée à l'écriture des femmes, et surtout à «l'écriture au féminin » de Nicole Brossard (p. 97).

L'intérêt de l'Espagne pour les écrits québécois ne date pas d'hier; une traduction en espagnol du roman Maria Chapdelaine a été publiée en 1923, soit sept ans après la première édition en français, et plusieurs rééditions ont suivi, en castillan et en catalan (p. 240).

Ouvrage bien structuré, Le Québec traduit en Espagne se subdivise en cinq chapitres portant sur les bases de la sociologie de la traduction, la 